\title{
Progesterone Induces Mucosal Immunity in a Rodent Model of Human Taeniosis by Taenia solium
}

\section{Galileo Escobedo1, Ignacio Camacho-Arroyo², Paul Nava-Luna ${ }^{3}$, Alfonso Olivos ${ }^{4}$, Armando Pérez-Torres ${ }^{5}$, Sonia Leon-Cabrera ${ }^{6}$, J.C. Carrero ${ }^{3}$ and Jorge Morales-Montor ${ }^{3 凶}$}

1. Unidad de Medicina Experimental, Hospital General de México, México D.F. 06726, México.

2. Facultad de Química, Departamento de Biología, Universidad Nacional Autónoma de México, México D.F. 04510, México.

3. Departamento de Inmunología, Instituto de Investigaciones Biomédicas, Universidad Nacional Autónoma de México, México D.F. 04510, México.

4. Departamento de Medicina Experimental, Facultad de Medicina, Hospital General de México, Universidad Nacional Autónoma de México, México D.F. 06726, México.

5. Departamento de Biología Celular y Tisular, Facultad de Medicina, Universidad Nacional Autónoma de México, México D.F. 04510, México.

6. Departamento de Microbiología y Parasitología, Facultad de Medicina, Universidad Nacional Autónoma de México, México D.F. 04510, México.

Corresponding author: Jorge Morales-Montor Ph.D. Departamento de Inmunología, Instituto de Investigaciones Biomédicas, Universidad Nacional Autónoma de México, AP 70228, México D.F. 04510, México. Telephone 0052(55)-5622-3854, Fax 0052(55) 5622-3369. E-mail: jmontor66@biomedicas.unam.mx

(C) Ivyspring International Publisher. This is an open-access article distributed under the terms of the Creative Commons License (http://creativecommons.org/ licenses/by-nc-nd/3.0/). Reproduction is permitted for personal, noncommercial use, provided that the article is in whole, unmodified, and properly cited.

Received: 2011.10.01; Accepted: 2011.10.26; Published: 2011.11.10

\begin{abstract}
More than one quarter of human world's population is exposed to intestinal helminth parasites. The Taenia solium tapeworm carrier is the main risk factor in the transmission of both human neurocysticercosis and porcine cysticercosis. Sex steroids play an important role during $T$. solium infection, particularly progesterone has been proposed as a key immunomodulatory hormone involved in susceptibility to human taeniosis in woman and cysticercosis in pregnant pigs. Thus, we evaluated the effect of progesterone administration upon the experimental taeniosis in golden hamsters (Mesocricetus auratus). Intact female adult hamsters were randomly divided into 3 groups: progesterone-subcutaneously treated; olive oil-treated as the vehicle group; and untreated controls. Animals were treated every other day during 4 weeks. After 2 weeks of treatment, all hamsters were orally infected with 4 viable $T$. solium cysticerci. After 2 weeks post infection, progesterone-treated hamsters showed reduction in adult worm recovery by $80 \%$, compared to both vehicle-treated and non-manipulated infected animals. In contrast to control and vehicle groups, progesterone treatment diminished tapeworm length by $75 \%$ and increased proliferation rate of leukocytes from spleen and mesenteric lymph nodes of infected hamsters by 5 -fold. The latter exhibited high expression levels of IL-4, IL-6 and TNF- $\alpha$ at the duodenal mucosa, accompanied with polymorphonuclear leukocytes infiltration. These results support that progesterone protects hamsters from the $T$. solium adult tapeworm establishment by improving the intestinal mucosal immunity, suggesting a potential use of analogues of this hormone as novel inductors of the gut immune response against intestinal helminth infections and probably other bowel-related disorders.
\end{abstract}

Key words: Taenia solium, cysticercosis, sex hormones, progesterone, inflammation.

\section{Introduction}

Taenia solium is a cestode parasite that affects both human and pigs $[1,2]$. Typically described as a health problem in developing countries $[3,4]$, T. so- lium infection has received growing attention to be considered as an emerging health problem in the United States and other developed countries $[5,6]$. 
The intermediate stage of T. solium causes human neurocysticercosis (hNC) and porcine cysticercosis [7]. hNC represents the most severe clinical manifestation of the human disease, whilst porcine cysticercosis is a source of enormous economic losses due to confiscation of contaminated swine meat $[4,8]$. The intestinal adult form of the parasite is receiving rising interest since it is considered as the main risk factor in the propagation of the disease for both organisms [9-11]. The fact that the T. solium tapeworm carrier be estimated as a central node in the maintenance of the infection is supported by the presence of $\mathrm{hNC}$ in human communities which do not consume swine meat, or even have no contact with pigs $[12,13]$. Then, more experimental and clinical research strategies should be directed to control the $T$. solium adult tapeworm establishment and egg production.

Sex steroid hormones have an important role during parasite infections [14-16], either by modulating host immune response [17-18] or having direct effects upon parasites [15]. Interestingly, pregnancy in female pigs and castration in male boars increase the prevalence of naturally acquired cysticercosis [19]. Moreover, T. solium infection decreases testosterone levels in non-castrated ranging boars [20]. This evidence leads us to assume that sex hormones can be either permissive or restrictive factors in the establishment of the intermediate stage of $T$. solium. In humans, intestinal taeniosis is more frequent in women than in men [21-23]. Similarly, the inflammatory response associated to the presence of brain located-cysticerci, exhibits a dimorphic pattern, being more severe in women than in men, presumably due to the higher number of eosinophils, and other proinflammatory efector cells, as well as IL-5 and IL-6 levels, in women cerebral spinal fluid [24].

In the same sense, female mice are more susceptible than males to experimental murine cysticercosis by T. crassiceps [25]. Such difference in susceptibility is abolished by gonadectomy of both genders [26]. However, restitution of progesterone to gonadectomized mice from both sexes decreased parasite loads by $100 \%$, presumably due to over-regulation of the intracellular progesterone receptor (PR) at splenic $\mathrm{T}$ lymphocytes [27] Similarly, ovariectomy of female mice exacerbates Trypanosoma cruzi parasitemia, meanwhile progesterone replacement drops it to similar levels as in control intact groups [28]. In a similar manner, Schistosoma haematobium-infected hamsters show a decrease in the number of recovered worms and egg load in response to the administration of medroxyprogesterone acetate, a progesterone analogue indicated as human contraceptive [29]. Pregnancy seems to have a protective role against Trichi- nella spiralis infection, attributed to the toxicity properties of progesterone against helminth [30]. As it can be seen, progesterone is strongly involved in the protection or susceptibility to several parasite infections, as it could be the case for the T. solium natural disease.

Complementary, progesterone also has several immunomodulatory actions [31]. For instance, it is directly involved in the immune tolerance against fetus presumably due to its influence on the activity of $\mathrm{T}$ cells and natural killer cells during pregnancy [31]. Additionally, progesterone is able to increase the synthesis of Th2-related cytokines such as IL-4 and IL-13, diminishing concomitantly the Th1-immune response mainly characterized by IL-12 and IFN- $\gamma$ expression [32]. Regarding to the $\mathrm{B}$ and $\mathrm{T}$ cells function, progesterone induces production of IgG class 1 antibodies and promotes the increase in the TCR gamma delta positive cell population [33]. This hormone is able to stimulate the differentiation of dendritic cells from healthy human-derived peripheral blood mononuclear cells which could be involved in the Th2-immune response polarization during pregnancy [34]. Then, besides the role of progesterone in parasitic diseases, it is clear that this hormone has the ability to influence the immune system by affecting cellular differentiation, cytokines and antibodies production, and effector cells activity [31-34].

Taking into consideration that progesterone can specifically modulate the immune response [35-38] and directly affect both cysticerci from $T$. solium and $T$. crassiceps $[39,40]$, it is possible that this hormone exerts a major role during the $T$. solium in vivo infection, by regulating the local immune response against this intestinal parasite.

Based on the evidence presented above, and for being considered one of the most important circulating hormones during female pregnancy with well described immunostimulator actions [41-45], here we evaluated the effect of progesterone upon the experimental taeniosis in golden hamsters (Mesocricetus auratus), having special emphasis in its influence upon the host mucosal immune response at bowel level.

\section{Materials and Methods Ethics Statement}

Animal care and experimentation practices at the Instituto de Investigaciones Biomédicas are frequently evaluated by the Institute's Animal Care and Use Committee, according to the official Mexican regulations (NOM-062-ZOO-1999). Mexican regulations are in strict accordance with the recommendations in the Guide for the Care and Use of Laboratory Animals of the National Institutes of Health (NIH and The 
Weatherall Report) of the USA, to ensure compliance with established international regulations and guidelines. The protocol was approved by the Committee on the Ethics of Animal Experiments of the Instituto de Investigaciones Biomédicas (Permit Number: 2009-16). Pigs sacrifice to obtain parasites was performed under sodium pentobarbital anesthesia, and all efforts were made to minimize suffering.

\section{Parasites}

T. solium cysticerci were selected according to the macroscopic criteria reported by León-Cabrera and coworkers [46]. Briefly, parasites were dissected from muscle of naturally infected pigs, which were euthanized at the Veterinary School of the Universidad Nacional Autónoma de México, under consent of the University Animal Care and Use Committee to ensure compliance with international regulations and guidelines. The fibrous capsule surrounding each parasite was carefully separated under a dissection microscope. Once separated, cysticerci were placed in tubes containing sterile PBS (1X) supplemented with 100 $\mathrm{U} / \mathrm{ml}$ of antibiotics-fungizone (Gibco,Grand Island, NY). Samples were centrifuged at $1200 \mathrm{rpm} / 4^{\circ} \mathrm{C}$ for $10 \mathrm{~min}$ and the supernatant was discarded. Pellets containing cysticerci were placed in Dulbecco`s Modified Medium (DMEM, Gibco, BRL, Rockville, MD) without fetal serum supplementation. Then, they were washed and centrifuged 3 times at 1200 $\mathrm{rpm} / 4^{\circ} \mathrm{C}$ for $10 \mathrm{~min}$. After the final wash, complete and translucent reddish cysticerci were incubated on 6-well culture plates containing DMEM medium with $25 \%$ pig fresh bile supplementation for infectivity test. When the evagination rate was higher than $90 \%$, then parasites were used for subsequent oral infections.

\section{Progesterone administration}

Ten female golden hamsters (Mesocricetus auratus) of 140-160 g, aging between 8 and 10 weeks, were subcutaneously administered with $2 \mathrm{mg} / \mathrm{Kg}$ body weight of water-soluble progesterone (Sigma-Aldrich, USA). Each single dose of progesterone was diluted in $0.4 \mathrm{~mL}$ of saline solution $(0.9 \% \mathrm{NaCl}$, Baker). Control animals $(n=10)$ received $0.4 \mathrm{~mL}$ of saline solution as vehicle. A stress-related additional control group $(n=10)$ was included in our experiments, which consisted in ten sham injection animals. Hormone and vehicle administration was carried out each other day during four weeks, in order to maintain the same hormonal serum concentration for the entirely time of the experiment. Our results were obtained from two independent experiments performed in similar conditions. Animals were fed with Purine
Diet 5015 (Purine, St. Louis, MO) and water ad libitum during all the experiment.

\section{Oral infection experiments}

Two weeks after the beginning of progesterone or vehicle administration, treated and untreated animals were orally infected with four viable T. solium cysticerci, according to previous reports [46]. All animals were euthanized 15 days post infection, using a $\mathrm{CO}_{2}$-saturated chamber. During animal necropsy, the entire small intestine was dissected and placed on a Petri dish containing sterile PBS (1X) (Sigma-Aldrich, USA). Under a stereoscopic microscope, the lumen of all small intestines was carefully exposed by making a longitudinal cut using sterile dissection scissors. Then, duodenum-anchored parasites were counted and measured with a calibrator. Blood samples were individually collected from all animal groups for posterior serum analysis. Ileum attachment zones where $T$. solium scolices were located, were placed in $4 \%$ paraformaldehyde (J.T. Baker, México), or Trizol reagent (Invitrogen, Carlsbad, California) for posterior analysis. Immediately after necropsy, spleen weight was individually recorded. Spleen samples and mesenteric lymph nodes from all animal groups were individually obtained and placed in RPMI (Gibco, BRL, Rockville, MD) supplemented with $10 \%$ fetal calf serum (Gibco, BRL, Rockville, MD), or 4\% paraformaldehyde (J.T. Baker, México), or Trizol reagent (Invitrogen, Carlsbad, California) for posterior analysis.

\section{Cell culture and lymphoid proliferation}

Total leukocytes and red blood cells were extracted from spleen and mesenteric lymph nodes of all animal groups. After single washing with ACK Lysing Buffer (Invitrogen, USA), total leukocytes were recovered and cultured in 96-well sterile plates $\left(1 \times 10^{4}\right.$ cells/well) containing serum-free RPMI medium (Gibco-BRL), at $37^{\circ} \mathrm{C}$ in humidified $5 \% \mathrm{CO}_{2}$ atmosphere for $72 \mathrm{~h}$. After this time, cultured leukocytes from spleen and mesenteric lymph nodes of all animals were exposed to $15 \mu \mathrm{g} /$ well of freshly extracted $T$. solium total antigen during $48 \mathrm{~h}$. Twenty four $\mathrm{h}$ before the end of the experiment, $20 \mu \mathrm{L}$ of AlamarBlue reagent (Biosource International) were added to each culture well. Then, culture plates were frozen at $-30^{\circ} \mathrm{C}$ under darkness and the absorbance was quantified at 570 and $600 \mathrm{~nm}$, using a microplate reader. The 570-600 nm lecture coefficient was employed to assess proliferation index.

\section{Cytokines and progesterone receptor expres- sion}

Spleen and anchored tapeworms-related duo- 
denum samples were placed in Trizol reagent (Invitrogen, Carlsbad, California). Total RNA extraction was as follows: spleen and duodenum were disrupted in Trizol reagent $(1 \mathrm{ml} / 0.1 \mathrm{~g}$ organ $)$ and $0.2 \mathrm{ml}$ of chloroform was added per $\mathrm{ml}$ of Trizol. The aqueous phase was recovered after $10 \mathrm{~min}$ of centrifugation at $13000 \mathrm{rpm}$, RNA was precipitated with isopropyl alcohol, washed with $75 \%$ ethanol and dissolved in RNAase-free water. RNA concentration was determined by absorbance at $260 \mathrm{~nm}$ and its purity was verified after electrophoresis on $1.0 \%$ denaturing agarose gel in presence of $2.2 \mathrm{M}$ formaldehyde. Immediately, total RNA samples were reverse-transcribed by using M-MLV Retrotranscriptase system and dT primer (Invitrogen, USA). Then, cDNA was specifically amplified by semi-quantitative PCR, using TaqDNA polymerase (Biotecnologías Universitarias, UNAM, México) and hamster-specific primers to detect IFN $\gamma$, IL-12, IL-4, TNF- $\alpha$, IL-6, PR and 18S-ribosomal RNA (Table 1). Briefly, the $50 \mu \mathrm{l}$ PCR reaction included $10 \mu \mathrm{l}$ of previously synthesized cDNA, $5 \mu \mathrm{l}$ of 10X PCR-buffer (Perkin-Elmer, USA), 1 $\mathrm{mM} \mathrm{MgCl}, 0.2 \mathrm{mM}$ of each dNTP, $0.05 \mu \mathrm{M}$ of each primer, and 2.5 units of TaqDNA polymerase (Biotecnologias Universitarias, Mexico). After an initial denaturation step at $95^{\circ} \mathrm{C}$ for $5 \mathrm{~min}$, temperature cycling was as follows:

$95^{\circ} \mathrm{C}$ for $30 \mathrm{~s}$, from $51^{\circ} \mathrm{C}$ to $62^{\circ} \mathrm{C}$ (depending on primer sequence) for $45 \mathrm{~s}$ and $72^{\circ} \mathrm{C}$ for $45 \mathrm{~s}$ during 35 cycles. An extra extension step was completed at $72^{\circ} \mathrm{C} / 10 \mathrm{~min}$ for each gene. The $50 \mu \mathrm{l}$ of the PCR reaction were electrophoresed on $2 \%$ agarose gel, stained with ethidium bromide in the presence of a $100 \mathrm{bp}$ ladder as molecular weight marker (Gibco, BRL, NY). Relative expression rate of each amplified gene was obtained by optical density analysis (OD), using the 18S-ribosomal RNA as constitutive control.

\section{Histological examination of inflammatory in- filtrate}

Anchored tapeworms-related duodenum samples and several fragments of jejunum and ileum from progesterone and vehicle-treated groups, as well as sham injection-infected animals, were placed in $4 \%$ paraformaldehyde for 2 weeks (J.T. Baker, México) and subsequently...processed to paraffin embedding and to obtain $4 \mu \mathrm{m}$ tissue sections which were stained with hematoxylin-eosin (H\&E) and toluidine blue to demonstrate mast cells. Specimens were washed twice with 1X PBS solution (Sigma-Aldrich, USA), and then were dehydrated using $70 \%, 80 \%, 95 \%$ and $100 \%$ ethanol (J.T. Baker, México), during 15 min-each one. Subsequently, organs were placed into xylene (J.T. Baker, México) for $30 \mathrm{~min}$ and embedded in $60^{\circ} \mathrm{C}$ liquid paraffin during $30 \mathrm{~min}$. After this time, liquid paraffin was replaced with new fresh $60^{\circ} \mathrm{C}$ paraffin. Once the blocks were solidified after $24 \mathrm{~h}$ at room temperature, duodenum tissue was cross-sectioned in thin $4 \mu \mathrm{M}$ slices, by using a microtome (Microtome Olympus Cut 4060, USA). Sections were stained with hematoxylin-eosin (H\&E). Evaluation of inflammatory infiltrate degree on H\&E-stained duodenum sections was performed under a light microscope using $400 \times$ magnification.

Table I. Primers used for amplification of hamster-specific genes. Primer sequences were designed based on hamster-specific gene sequences reported in the Gene databank, NCBI, NIH. Primer sequence as well expected molecular weight of the $P C R$ product is shown. $b p=$ base pairs.

\begin{tabular}{|c|c|c|}
\hline $\begin{array}{l}\text { Primer defini- } \\
\text { tion }\end{array}$ & Primer sequence & $\begin{array}{l}\text { Molecular } \\
\text { weight of } \\
\text { the PCR } \\
\text { product } \\
\text { (bp) }\end{array}$ \\
\hline IFN- $\gamma$ (sense) & 5'-CAAAAGGCTGGTGACACAAA & \multirow{2}{*}{326} \\
\hline IFN- $\gamma$ (antisense) & 5'-TTCTTGTTGGGACGATTTCC & \\
\hline IL-12 (sense) & 5'-CTCTGAGCCACTCACGA & \multirow{2}{*}{167} \\
\hline IL-12 (antisense) & 5'-GTCAGTGCTGATTGCA & \\
\hline IL-4 (sense) & 5'-CCAGGTCACAGAAAAAGGGA & \multirow{2}{*}{247} \\
\hline IL-4 (antisense) & 5'-CGTGGACTCATTCACATTGC & \\
\hline IL-6 (sense) & 5'-CAACAAGTCGGAGGTTTGGT & \multirow[b]{2}{*}{302} \\
\hline IL-6 (antisense) & 5'-AGGGTTTTGATGGTGCTCTG & \\
\hline TNF- $\alpha$ (sense) & 5'-GGGAAGAGAAGTTCCCCAAC & \multirow[b]{2}{*}{229} \\
\hline $\begin{array}{l}\text { TNF- } \alpha \text { (anti- } \\
\text { sense) }\end{array}$ & 5'-TAAACCAGGTACAGCCCGTC & \\
\hline PR (sense) & 5'-GGAGGCAGAAATTCCAGACC & \multirow[b]{2}{*}{198} \\
\hline PR (antisense) & 5'-GACAACAACCCTTTGGTAGC & \\
\hline $18 \mathrm{~S}$ (sense) & 5'-CGCGGTTCTATTTTGTTGGT & \multirow[b]{2}{*}{219} \\
\hline $18 \mathrm{~S}$ (antisense) & 5'-AGTCGGCATCGTTTATGGTC & \\
\hline
\end{tabular}

\section{Immunofluorescense}

Antibodies anti-mouse IL2, IL1 $\beta$, IL5 and TGF $\beta$ done in rabbit, anti-mouse IFN $\gamma$, TNF $\alpha$, IL12, IL10, IL6 and IL13 done in goat, and anti-mouse IL4 done in rat were used as primary antibodies (Santa Cruz, Biotecnology, USA). Anti-rabbit IgG and anti-goat IgG conjugated with rhodamine (TRITC) and anti- rat IgG conjugated with fluorescein isothiocyanate (FITC) (ZIMED Laboratories Inc., USA) were used as secondary antibodies. Duodenums were fixed in $4 \%$ paraformaldehyde for $48 \mathrm{~h}$, washed with PBS and stored in PBS containing $30 \%$ sucrose at $4^{\circ} \mathrm{C}$ overnight. Next day, samples were embedded in tissue-freezing medium (Leica, Nussloch, Germany) and frozen at $-70^{\circ} \mathrm{C}$ (dry ice hexane). Serial sections of 20 $\mu \mathrm{m}$ thicknesses were obtained using a cryomicrotome, placed on slides coated with poly-L-lysine (Sigma) 
and air-dried. Sections were treated with $1 \%$ Triton X-100 after blocking with $1 \%$ albumin (BSA) and incubated with primary antibody overnight at $4^{\circ} \mathrm{C}$ diluted in 1:1000 BSA-PBS 1X. After rinsing in PBS, the sections were incubated with the secondary antibody during $1 \mathrm{~h}$ at room temperature diluted in 1:200 BSA-PBS $1 \mathrm{X}$, washed in PBS and embedded in anti-fading DAKO mounting medium (DAKO, USA). Intestine sections processed without the primary antibody were used as negative controls.

For each group, several microscopic fields of duodenum were captured and analyzed by semiquantitative inmunofluorescence laser confocal microscopy. In brief, TIFF images were acquired with the TCS-SP1 software and imported into Image Pro Plus for subsequent measuring of the immunofluorescence intensity. In each image, representative areas were selected and, with the exposure times kept constant, the intensity of fluorescence was quantified and expressed as the mean pixel intensity for each region. For each animal, at least six randomly selected areas were analyzed.

\section{Experimental design and statistical analysis}

Our results were estimated in 2 independent experiments. All experimental groups consisted in 10 animals each. Dependent variables were number of intestinally attached parasites, adult tapeworm length $(\mathrm{mm})$, proliferation index, IL-4, IL-6, TNF- $\alpha$ and PR expression, as well number of inflammatory foci. Number of mast cells is expressed at $\mathrm{mm}^{2}$ of tissue section. The independent variable was progesterone treatment. Data from 2 replicates $(n=20)$ of each experimental group were expressed as an average +/standard deviation, and analyzed by means of one way-ANOVA, and Tukey test as post hoc test. Differences were considered significant when $\mathrm{P}<0.05$.

\section{Results}

After 15 days post infection, progesterone treatment significantly reduced the number of intestinally anchored-T. solium tapeworms by $80 \%$ (Fig. 1A). Vehicle-treated and sham injection-infected hamsters showed between three and four viable parasites (Fig. 1A). It is important to remark that all found tapeworms were strongly attached to the duodenum zone. As expected, tapeworms from vehicle-treated and sham injection-infected hamsters grew up more than $1.5 \mathrm{~mm}+/-0.4$ (Fig. 1B). In contrast, parasite from progesterone-treated hamsters did not develop more than $0.2 \mathrm{~mm}+/-0.16$ in length (Fig. 1B), showing besides poorly differentiated scolices. Thus, steady concentrations of progesterone exerted a protective role against the $T$. solium intestinal infection, diminishing both the number of attached parasites and their development.

To assess the possible mechanism involved in progesterone protective actions during infection, spleens from all animal groups were weighed and splenic leukocytes assayed for antigen-specific proliferation (Fig. 2). Although no significant differences were observed among the spleen weight from progesterone, vehicle-treated animals and sham injection-infected hamsters (Fig. 2A), there was a discrete but interesting tendency in hamsters exposed to progesterone to increase spleen weight respect to their infected control littermates. Furthermore, in vivo progesterone treatment clearly increased proliferation in vitro of $T$. solium antigen-specific leukocytes by 5-fold compared to both infected control groups (Fig. 2B). This result suggests that progesterone should protect hamsters from $T$. solium infection through promotion of a local mucosal anti-parasite immune response.

To evaluate the immuno-stimulating effects of progesterone, cytokine profile expression from spleen and parasite associated-duodenum, as well as PR expression, were assessed (Fig. 3). In spleen, progesterone did not modify gene expression of IL-12 and IFN- $\gamma$ (Figs. 3A, 3B) but significantly increased by 8 -fold the expression of IL-4, one of the most representative Th2-related cytokines (Fig. 3E). A similar effect was observed in the expression of proinflammatory cytokines IL- 6 and TNF- $\alpha$, which showed an increase by 5-fold and 9-fold, respectively (Figs. 3C, 3D). Of special interest was to determine the cytokines profile of duodenal mucosa from progesterone-treated and untreated hamsters, because this tissue was in direct contact with parasites. Interestingly, both proinflammatory cytokines IL-6 and TNF- $\alpha$ were locally increased by 6-fold each (Figs. 3H, 3I), as well as IL-4 (Fig. 3J), in response to progesterone treatment. As was seen in spleen, duodenal expression of IL-12 and IFN- $\gamma$ was not modified by any treatment (Fig. 3F, 3G). PR expression in progesterone-treated animals was diminished by 4-fold in the spleen, compared to both control infected groups (Fig. 4A). Moreover, progesterone treatment also down-regulated PR expression by $50 \%$ in tapeworms-associated duodenum (Fig. 4B). We speculate that progesterone induces protection on $T$. solium-infected hamsters by locally increasing proinflamatory and Th2-related cytokines expression, probably mediated by its intracellular PR, which showed a classical down-regulation pattern in response to progesterone. 

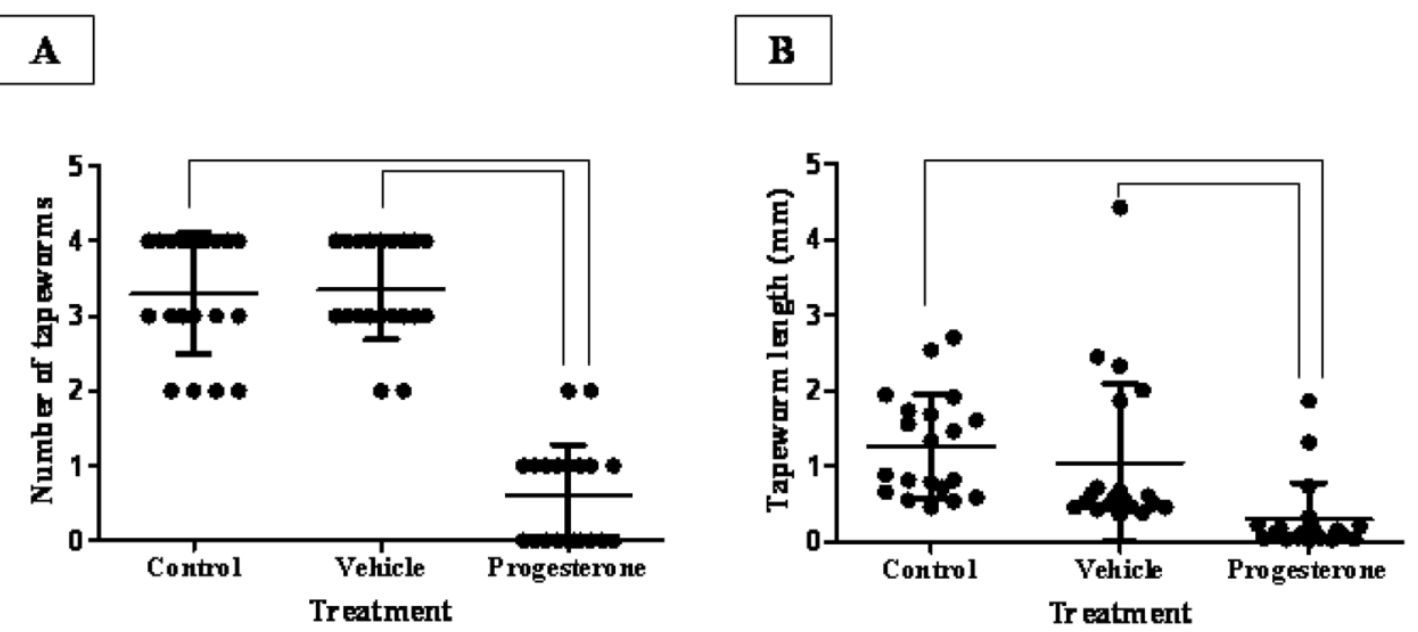

Figure I. Progesterone decreases both parasite load and tapeworm length in T. solium cysticerci-orally infected hamsters. (A) Administration of progesterone significantly diminished the number of intestinally attached adult tapeworms by $80 \%$, respect to both control and vehicle infected groups. (B) Parasites exposed to constant concentrations of progesterone showed total length reduction of seven-fold. These parasites seemed as undifferentiated scolices with no develop of neck and strobila, compared to those tapeworms from control and vehicle groups with well differentiated structures. Tapeworm length was determined as the longitudinal sum of scolex, neck and strobila. Non-manipulated infected hamsters were denominated as Control, meanwhile comparative lines represent significant differences when $P<0.05$. Results are presented as mean $+/$ - standard deviation.

\section{$\mathbf{A}$}

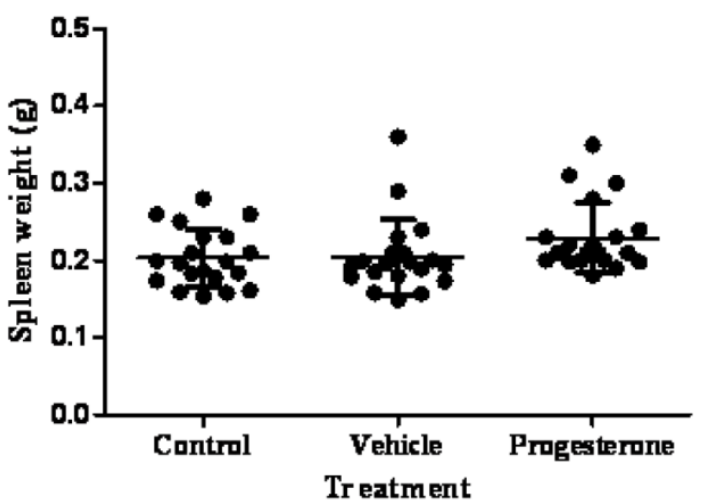

B

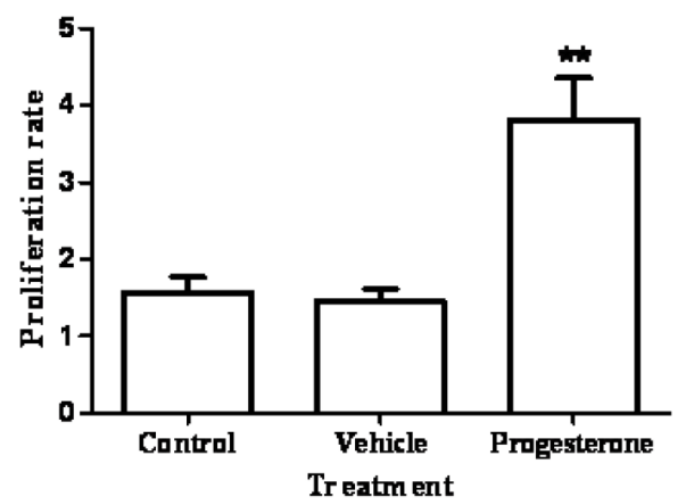

Figure 2. Progesterone administration increases proliferation rate of parasite specific-leukocytes from spleen and mesenteric lymph nodes. (A) Although progesterone-treated animals showed a discrete tendency to increase spleen weight, no significant differences in spleen weight were observed among treatments. (B) Spleen and mesenteric lymph nodes leukocytes from progesterone and vehicle-treated, as well non-manipulated infected hamsters were in vitro cultured in presence of $T$. solium total antigen. As a consequence of the in vivo exposition to progesterone, proliferation index of parasitic antigen specific-leukocytes was augmented by 3.5 and 5 -fold, respect to immune cells from both control and vehicle-treated hamsters. Non-manipulated infected hamsters were denominated as control. Results are presented as mean $+/$ - standard deviation. ${ }^{* * P}<0.05$ vs the other groups. 

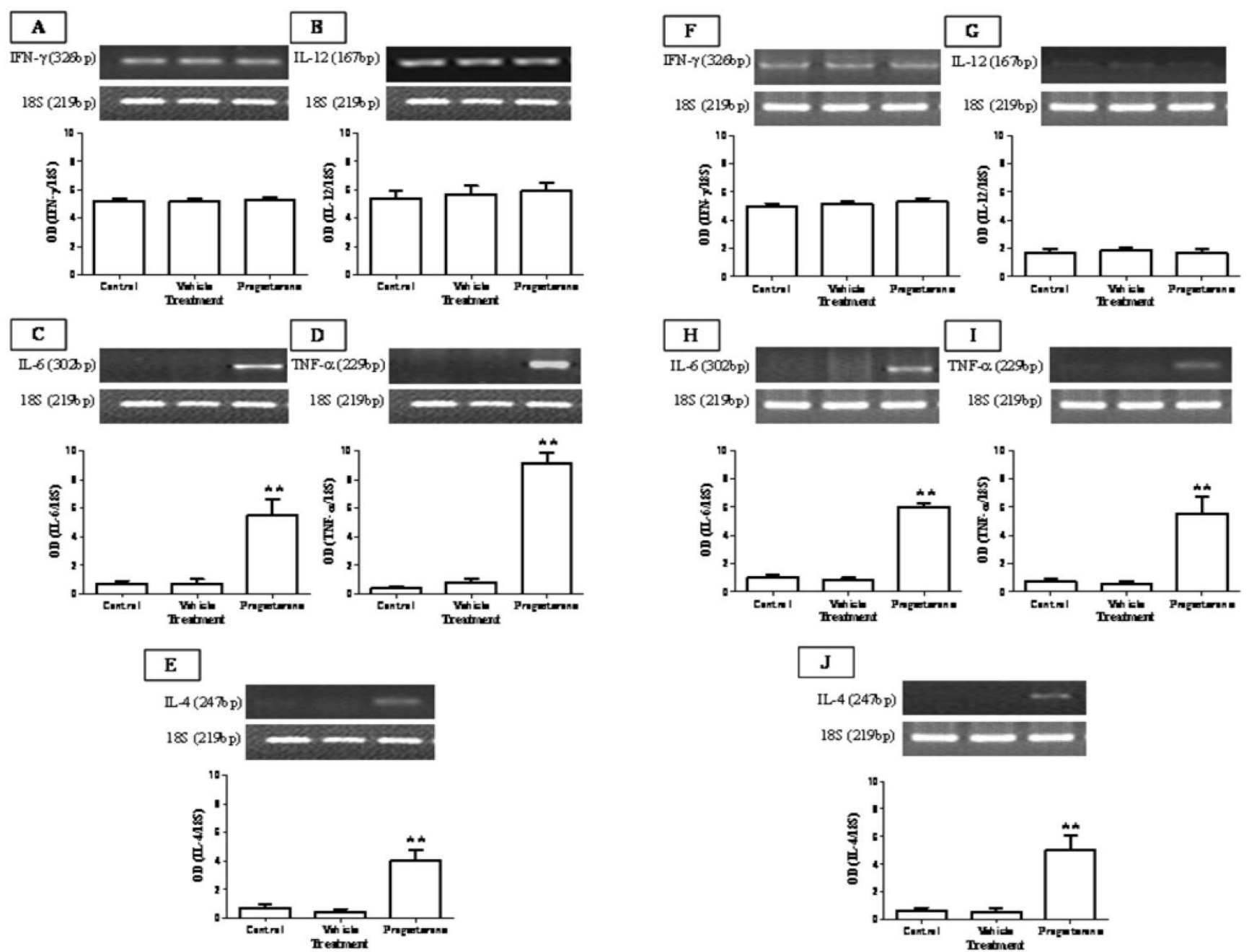

Figure 3. Progesterone administration induces IL-6, TNF- $\alpha$ and IL-4 expression on $T$. solium cysticerci-orally infected hamsters. Progesterone induced expression of proinflamatory and Th2-related cytokines IL-6, TNF- $\alpha$ and IL-4, systemically from spleen (A-E) and locally on parasite-associated duodenum (F-J). No significant effects were observed in the Th I-related cytokines IFN- $\gamma$ and IL-12 expression. 18S was used as control gene of constitutive expression. Optical densitometry (OD) was obtained as a relation between the genes of interest and I8S, and reported as mean $+/$ - standard deviation. $* * P<0.05$ vs the other groups.

Figure 4. Progesterone receptor (PR) expression is reduced in response to progesterone administration. PR showed a classical down-regulation in (A) spleen and (B) duodenum of progesterone-treated infected hamsters. In the spleen, PR expression was diminished by 4 -fold respect to both control and vehicle groups $(A)$, meanwhile it presented a decrease of 5 -fold in parasite-associated duodenum (B). Data are reported as mean +/- standard deviation. $* * P<0.05$.

\section{A}
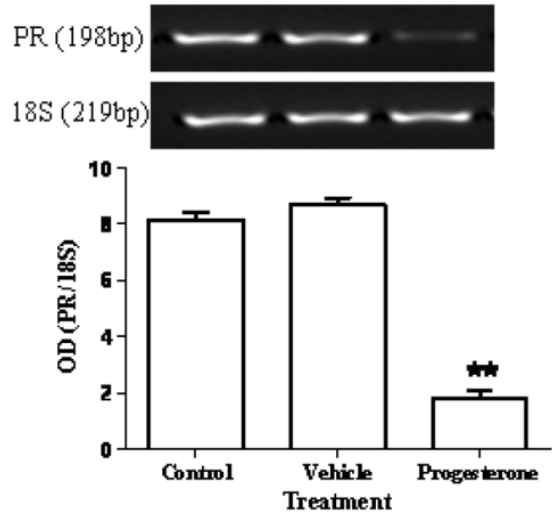

B
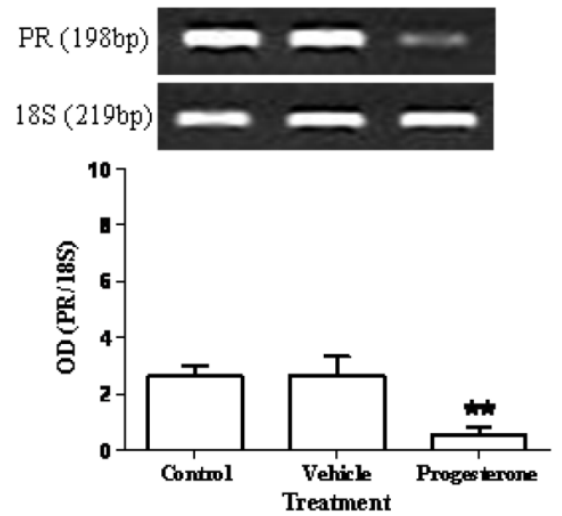
Local inflammation at intestinal sites where tapeworms were found was evaluated (Fig. 5). Histological condition of duodenal mucosa from control infected animals was quietly normal, exhibiting a well-defined intestinal villi formed by tunica mucosa. (Fig. 5A, 5B). Progesterone-treated hamsters presented an exacerbated inflammatory infiltrate located along the lamina propria, clearly related to the parasite presence, mainly characterized by eosinophils $(\sim 80 \%)$, lymphocytes $(\sim 10 \%)$ and basophils $(<5 \%)$ (Fig. 5C, 5D). Unexpectedly, bigger and reddish Peyer's patches were observed in this experimental group, and when parasites were closely located to these immune structures, they exhibited more damage and poor differentiation. As a consequence of such inflammation intensity (Fig. 5D), several properties of the duodenal tissue were lost, such as lack of microvilli number and enlargement, as well as an important increase in goblet cells and mucus production (Fig. 5C). In vehicle-treated (Fig. 5B) and sham injection- infected animals (Fig. 5A) there was an apparent inflammatory infiltrate in response to the presence of the parasite. However, such inflammation reaction had no the same magnitude than such observed in progesterone-treated animals (Fig. 5D), which probably was strongly related to differences in number of attached parasites, their appearance and length from both progesterone-treated and untreated studied groups.

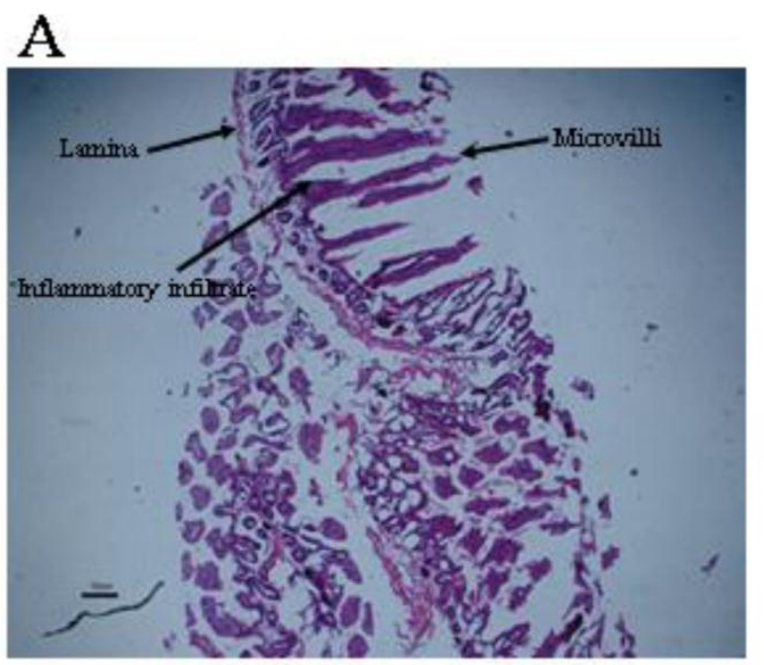

\section{B}
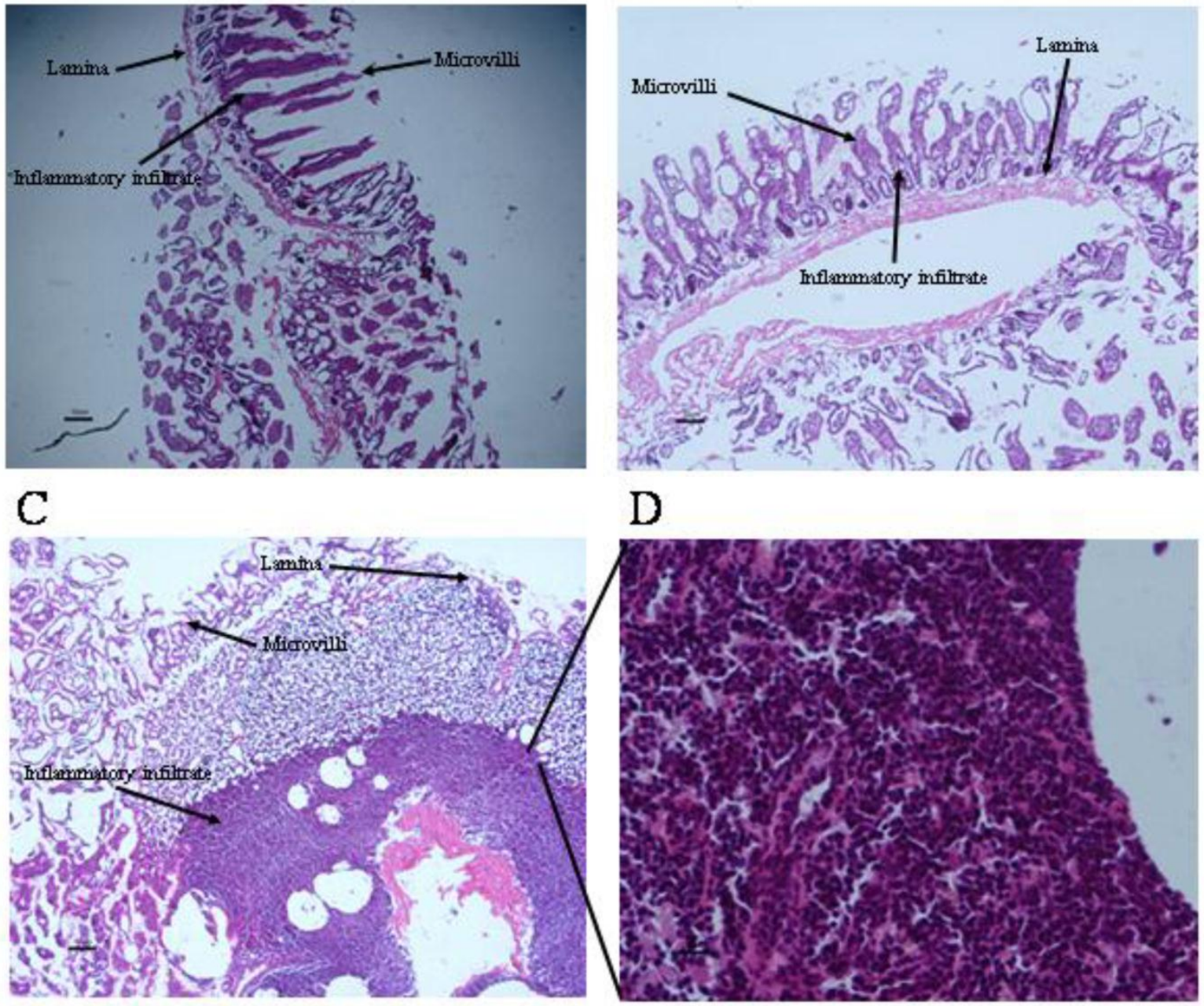

Figure 5. Progesterone promotes exacerbation of inflammatory infiltrate on parasite-associated duodenum from infected hamsters. (A) Non-manipulated infected hamsters and (B) vehicle infected hamsters showed no exacerbated inflammatory infiltrate and well defined tissue structures, although presence of inflammatory infiltrate associated to parasites was still evident. (C) Progesterone treated and infected hamsters showed an exacerbated inflammatory response at duodenal mucosa with eosinophils, basophils and lymphocytes infiltration. (D) Progesterone treated and infected hamsters showed as well lack of tissue arrangement. A: 6X magnification. B-C: I0X magnification. D: 40X magnification. Scale bar=50 microns. 


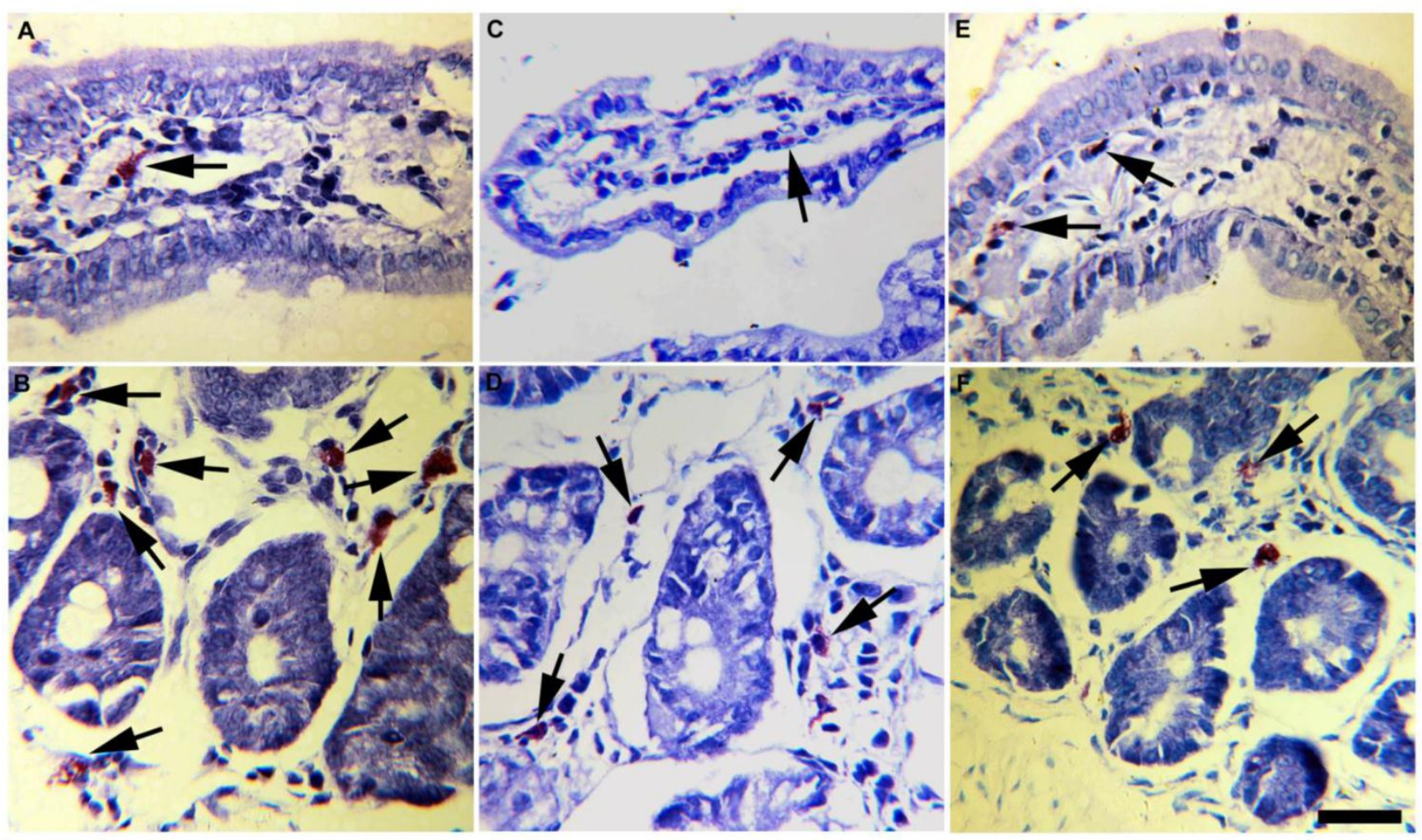

Figure 6. Mucosal mast cells from small intestine mucosa of experimental T. solium-infected hamsters. Progesterone-treated and infected hamsters (A, B) contained more mast cells with abundant and bigger metachromatic granules in the cytoplasm, than vehicle-treated and infected hamsters (C, D) and non-manipulated infected-hamsters (E, F). These findings were consistently observed in mast cells located either in periglandular lamina propria (B, D, F) as in intestinal villi (A, $C, E)$. Arrows indicate toluidine blue stained mast cells. Scale bar $=25 \mu \mathrm{m}$.

Accordingly, progesterone administration induced infiltration of mast cells mainly located at the lamina propria of intestinal glands (Fig. 6B) and villi (Fig. 6A). In progesterone-treated animals mast cells infiltrate was closer associated to parasite's attachment zones and Peyer's patches. Moreover, these cells were bigger than those observed in the small intestine of vehicle (Figs. 6 C,D) and control (Figs. 6 E,F) hamsters, exhibiting high rate of metachromatic granules. A higher number of mononuclear and plasmatic cells were frequently observed in these intestinal specimens. In contrast, vehicle and control animals showed low number of small mast cells with poor degranulatory activity (Figs. 6C-F). Overall, parasite elimination was strongly associated with intestinal tissue damage which in turn showed high correlation with progesterone-induced mast cells degranulation. These data support that progesterone administration is able to exacerbate the host's mucosa inflammatory response that subsequently could mediate reduction in parasite number and growth.

As the anti-parasite immune response in hamsters was related with local inflammation, we carried out immunohistochemical assays in tissue sections from duodenum, in order to determine and compare the local profile of cytokines in each studied group. The mean intensity of IFN- $\gamma$ was significantly augmented in duodenum sections from progesterone-treated animals, as compared to vehicle-treated and untreated controls (Table 2). Concomitantly, production of IL-5 and IL-13 was diminished in response to the administration of this hormone. This group also exhibited a decrease in the mean intensity of TNF- $\alpha$, whilst fluorescence intensity related to IL-10 was clearly increased by 4 -fold concerning controls. These results suggest that progesterone is able to modify the immunological profile of cytokines at the intestinal mucosa of hamsters, which could be associated with protection against the $T$. solium infection.

\section{Discussion}

Several efforts have been performed in order to control helminthiasis such as onchocerciasis, filariasis, schistosomiasis and taeniosis, among others [47-50]. Mostly based on drug and vaccine design [11, 46, 47, 51], these strategies focus on inhibiting establishment 
and reproduction of adult intestinal worms. Although the adult stage of most of these parasites does not cause any severe pathology, with practically asymptomatic clinical manifestations [52], it is now recognized as the main risk factor in the propagation of the disease to natural and accidental intermediate host $[10,11,46]$. It is the case for human and porcine cysticercosis by $T$. solium $[10,46]$. For this reason and by using the experimental taeniasis model in hamsters, our work centers to evaluate the possible protective role of progesterone upon the establishment of the $T$. solium adult tapeworm, since this hormone possess immunomodulatory properties and participates in pig cysticercosis [27-30].
In fact, progesterone showed a protective effect upon establishment of the T. solium adult stage in hamsters. It is important to mention that the administration of progesterone significantly reduced parasite load and tapeworm growth, with similar results to those obtained through vaccination [46, 51-53]. Therefore, combined treatment of immunogenic molecules and progesterone could be a hopeful alternative for reach a total protection against intestinal parasites invasion. In regard to this issue, several studies report the use of hormones as vaccine's adjuvants with prominent results preventing type A influenza, tetanus and tuberculosis [54-57].

Table 2. Cytokine pattern in duodenal tissue from progesterone-treated and control hamsters infected with the adult tapeworm of Taenia solium. ${ }^{a}$ Fluorescence intensity data are presented as mean \pm standard deviation. ${ }^{b} \mathrm{P}<0.05,{ }^{\mathrm{C}} \mathrm{P}<0.0 \mathrm{I}$.

\begin{tabular}{|c|c|c|c|c|}
\hline \multirow[b]{2}{*}{ Cytokine Family } & \multirow[b]{2}{*}{ Cytokine } & \multirow[b]{2}{*}{ Untreated controls ${ }^{a}$} & \multicolumn{2}{|l|}{ Groups } \\
\hline & & & Vehicle $^{a}$ & Progesterone \\
\hline \multirow{3}{*}{ Th1 } & IL-2 & $53.8 \pm 3.9$ & $56.2 \pm 4.2$ & $54.6 \pm 1.1$ \\
\hline & IL-12 & $50.4 \pm 4.6$ & $53.4 \pm 3.2$ & $52.5 \pm 3.8$ \\
\hline & IFN- $\gamma$ & $44.1 \pm 2.4$ & $46.6 \pm 4.1$ & $52.1 \pm 3.3^{b}$ \\
\hline \multirow{4}{*}{ Th2 } & IL-4 & $51.2 \pm 1.8$ & $50.5 \pm 0.6$ & $49.8 \pm 3.3$ \\
\hline & IL-5 & $44.6 \pm 1.5$ & $43.7 \pm 2.1$ & $32.8 \pm 3.3^{c}$ \\
\hline & IL-13 & $47.2 \pm 2.7$ & $44.0 \pm 3.6$ & $25.0 \pm 3.0^{c}$ \\
\hline & TGF- $\beta 1$ & $31.5 \pm 3.2$ & $30.0 \pm 3.0$ & $34.1 \pm 1.5$ \\
\hline \multirow{3}{*}{ Proinflammatory } & IL-1 $\beta$ & $45.9 \pm 3.6$ & $46.5 \pm 4.1$ & $46.2 \pm 2.7$ \\
\hline & IL-6 & $27.8 \pm 1.7$ & $28.4 \pm 1.2$ & $25.7 \pm 0.8$ \\
\hline & TNF- $\alpha$ & $54.1 \pm 2.4$ & $55.5 \pm 1.8$ & $42.6 \pm 2.8^{b}$ \\
\hline Regulatory & IL-10 & $8.6 \pm 0.9$ & $8.0 \pm 1.1$ & $32.6 \pm 6.1^{c}$ \\
\hline
\end{tabular}

Progesterone also seems to over-activate immune cells that specifically recognize parasite antigens. Worth of mention is the prominent proliferation rate of $\mathrm{T}$ lymphocytes in presence of $T$. solium antigens, which lead us to suppose that progesterone may act through two main ways: a) by increasing antigen specific-immune cell viability and proliferation or b) inducing a long duration-preactivated status on host immune cells. Although both possible explanations are intriguing, they require further experimental analysis.

On the other side, progesterone was able to locally over-regulate proinflammatory and Th2-related cytokines expression. As we have previously shown, the immunomodulatory effects of progesterone on several peripheral immune cell types have seen widely described [43, 45, 58, 59]. Nevertheless, its regulator role upon mucosal immunity has been scarcely studied [60-62]. Our data show that progesterone treatment has the ability to induce IL-4, IL-6 and TNF- $\alpha$ expression at duodenal mucosa of infected hamsters. Expression of these cytokines has been associated to parasite elimination in this animal model [63]. Then, progesterone could improve protection from $T$. solium infection by peripherally increasing parasite specific-immune cell proliferation, with concomitant local stimulation of IL-4, IL- 6 and TNF- $\alpha$ expression. In turn, these cytokines could promote an exacerbated inflammatory reaction with enormous capacity to affect parasite establishment and growth through the activation of eosinophil, basophil and, importantly, mast cells. Of additional interest is the possible direct role of progesterone upon these immune cell types associated to the mucosa. For in- 
stance, during human chronic urticaria several reports suggest that mast cells and eosinophil could be over-activated by pregnancy-related factors such as progesterone and estrogens [64,65]. Concomitantly, $17 \beta$-estradiol is able to promote release of $\beta$-hexosaminidase and IgE-induced degranulation from mast cell and basophil cell lines [66], meanwhile on uterine mast cells this sex steroid significantly increases histamine release [67]. This suggests that mucosa associated-innate immune response cells should be affected by sex steroid hormones [66, 67]. However, further investigation concerning the participation of these hormones in the modulation of mast cell, basophil and eosinophil activity at the intestinal mucosa is required.

Progesterone effects may be mediated by its intracellular PR. Our data show that PR expression was down-regulated by progesterone treatment, which denotes a classical feed-back regulation, previously described in several tissues as a regulatory mechanism of progesterone actions. However, to assess this point is necessary the use of anti-progestins with capacity to competitively bind to PR isoforms in hamsters $[68,69]$.

In experimental T. solium infection in hamsters, the acute inflammatory response is characterized by the presence of eosinophil, neutrophils, macrophages and lymphocytes, that promote destruction of the surrounding cells $[26,27,38]$. Even though Th1-type cytokines activate neutrophils and macrophages in vitro resulting in parasiticidal activity mediated by nitric oxide [39-41] experiments in vivo have shown that neutrophils and macrophages are not capable of killing taenias. The host's cellular immune response against parasites is regulated by cytokines, and therefore the Th1/Th2 type polarization can result in protection or susceptibility [43]. Several reports have described the ability of sex hormones to influence all cellular types of the innate and adaptive immune systems, thereby modifying a multitude of immunological functions [44, 45]. Moreover, sex hormones influence the development, maturation and state of activation of $\mathrm{T}$ lymphocytes, including the Th1/Th2 balance $[47,48]$.

It has been reported that in the late phase of $T$. solium infection in hamsters, when animals recover from an acute immunosuppression, a Th1 response occurred: IL-2 seems to be the major cytokine responsible for protection, whereas Th2-cytokines seem to be associated with susceptibility $[46,49,50]$. Our study sampled a broad profile of the cytokines with ability of influencing the course of infection in hamsters. At protein levels, semiquantitative immunofluorescence analysis from duodenum tissue samples suggested that the treatment with progesterone resulted in low levels of Th2-cytokines, accompanied by polarization of the cellular response toward a Th1-type, which has been related with parasite elimination $[46,49,50]$. In contrast to results obtained by mRNA expression, protein levels of TNF- $\alpha$ are significantly reduced in progesterone-treated animals, while IL-10 is dramatically increased. This apparently controversial result can be explained since gene products are susceptible of post-transcriptional regulation mechanisms, as it is well known. Such mechanisms guarantee a balanced response at protein and cellular levels. Particularly, IL-10 could be overproduced in order to diminish exacerbation of the inflammatory response at the duodenal mucosa, which besides eliminating taenias, could result in serious detriment for the host. Thus, the protective effect of the Th1-immune profile observed in progesterone-treated hamsters supports the notion that a strong Th1 response is associated with parasite elimination. Concomitantly, elevation of regulatory cytokines could be involved in preventing inflammation-related injury at the duodenal mucosa of the host.

Progesterone treatment could also have a direct effect on the parasite. In this regard, T. solium has the ability to modulate the expression of molecules related with virulence upon contact with host components, including hormones. In other parasites, specific hormone receptors have been shown to regulate the expression of diverse proteins [52, 53]. Our preliminary data indicate that a non-classic receptor for progesterone is present in the cysticerci of T. solium [40].

Although the hamster model used here possibly does not reflect exactly all aspects of human taeniosis, a set of interactions are established between the parasite, the immune system and the endocrine system, which define the outcome of the infection caused by T. solium. Evidence presented here shows that progesterone treatment in hamsters reduces establishment and development of T. solium, possibly due to a specific increase in the local mucosal immunity in the duodenum, characterized by a strong inflammatory infiltrate and a Th1 immune response, suggesting that interactions between the immune and endocrine systems play a fundamental role in the establishment, development and outcome of intestinal T. solium infection in hamsters.

It is important to say that the experimental proof exposed in this work does not only concern to intestinal parasite infections, but also to other highly prevalent human gastrointestinal disorders where hormones seems to play a decisive role [70-80] such as irritable bowel syndrome (IBS) characterized by recurrent abdominal pain, bloating and constipation 
which is one of the most frequent chronic pelvic pain around the world $[70,71]$. Besides, the evidence that progesterone and estradiol are able to modify the intestinal transit time, visceral sensitivity and gut function [72-74] it has been demonstrated that the administration of progesterone diminishes intestinal production of IL- $1 \beta$ and TNF- $\alpha$, decreasing in turn bowel inflammation, damage and apoptosis in rats undergoing traumatic brain injury [75]. Concomitantly, several clinical trials suggest that the use of medroxyprogesterone acetate (a progesterone analogue) exerts benefits for IBS treatment [71]. Additionally, Freedman and coworkers showed that higher levels of progesterone in mature women subjected to hormonal replacement therapy correlate with low incidence of esophagus and stomach adenocarcinomas, which may partially explain the higher prevalence rates of gastrointestinal tract cancers in men than in women [76]. In a similar way, a hormonal therapy based on the daily administration of ethynil estradiol $(30 \mu \mathrm{g})$ and noretisterone $(1.5 \mathrm{mg}$ ) (both analogue molecules of estradiol and progesterone, respectively) is able to decrease chronic bleeding associated to gastric antral vascular ectasia in decompensated cirrhotic patients [77]. Moreover, high-turnover type osteoporosis (a common complication in patients with primary biliary cirrhosis) is significantly attenuated by hormonal replacement therapy without increasing risk of cholestasis [78]. Progesterone also has beneficial effects on Helicobacter pylori-associated gastritis in female ovarectomized gerbils, which showed a significant reduction in gastrin-positive cells [79]. In female Sprague-Dawley rats, administration of progesterone $(300 \mu \mathrm{g} /$ rat $)$ reduced cysteamine-induced peptic ulcers apparently due to the property of this hormone to increase gastroduodenal mucus levels involved in long gastric protection [80]. Then, progesterone benefits are not only limited to improve a restrictive immune response against intestinal parasites, but it seems to have a major protective role during several human gastrointestinal pathologies, which highlights the possible use of progesterone and progesterone's analogues as a promissory alternative treatment for management of gastric and esophagus carcinomas, intestinal inflammatory disease, gastritis, peptic ulcer and parasite-related intestinal infections, among others.

The evidence presented in our work illustrates the importance of immunoendocrine interactions in an immunocompetent host. It strongly suggests an important role for sex steroids, particularly progesterone, in the cytokine network. The complexity of the immunoendocrine interactions suggests that all physiological factors (i.e., sex, age, developmental stage) should be taken into consideration in the design of vaccines and new drugs. Interventions aimed at the hormonal network appear as a possible new therapeutic approach to control several immune confrontations, such as taeniosis/cysticercosis, as well as other related enteric disorders.

Taking into consideration the feasible role of progesterone on mucosal immunity, present results may open an interesting perspective in the possible use of sex steroid hormone analogues as adjuvants for anti-parasite vaccination, with strong immune actions but minimal endocrine effects, which could contribute to design different strategies for control intestinal helminth infections such as taeniosis by T. solium and in a nearer future other human gastrointestinal diseases.

\section{Acknowledgments}

Financial support was provided by grant IN 214011-3 from the Programa de Apoyo a Proyectos de Innovación Tecnológica, Dirección General de Asuntos del Personal Académico, Universidad Nacional Autónoma de México to J. Morales-Montor. G. Escobedo thanks financial support from Fundación Mexicana para la Salud, A. C., Grant no. 573-Estímulo Antonio Ariza Cañadilla. S. Leon-Cabrera has a doctoral fellowship from CONACYT at the Programa de Doctorado en Ciencias Biomédicas, UNAM.

\section{Conflict of Interests}

The authors have declared that no conflict of interest exists.

\section{References}

1. Flisser A, Sarti E, Lightowlers M, et al. Neurocysticercosis: regional status, epidemiology, impact and control measures in the Americas. Acta Trop. 2003; 87: 43-51.

2. Garcia HH, Del Brutto OH. Neurocysticercosis: updated concepts about an old disease. Lancet Neurol. 2005; 10: 653-661.

3. Nash TE, Singh G, White AC, et al. Treatment of neurocysticercosis: current status and future research needs. Neurology. 2006; 67: 1120-1127.

4. Fan PC, Chung WC. Sociocultural factors and local customs related to taeniasis in east Asia. Kaohsiung J Med Sci. 1997; 13: 647-652.

5. White AC Jr. Neurocysticercosis: a major cause of neurological disease worldwide. Clin Infect Dis. 1997; 24: 101-113.

6. Esquivel A, Diaz-Otero F, Gimenez-Roldan S. Growing frequency of neurocysticercosis in Madrid (Spain). Neurologia. 2005; 20: 116-20.

7. Pawlowski Z, Allan J, Sarti E. Control of Taenia solium taeniasis/cysticercosis: from research towards implementation. Int J Parasitol. 2005; 35: 1221-1232.

8. Gonzalez AE, Gavidia C, Falcon N, et al. Protection of pigs with cysticercosis from further infections after treatment with oxfendazole. Am J Trop Med Hyg. 2001; 65: 15-18.

9. Behravesh CB, Mayberry LF, Bristol JR, et al. Population-based survey of taeniasis along the United States-Mexico border. Ann Trop Med Parasitol. 2008; 102: 325-333. 
10. Flisser A, Avila G, Maravilla P, et al. Taenia solium: current understanding of laboratory animal models of taeniosis. Parasitology. 2010; 137: 347-57.

11. Sciutto E, Rosas G, Cruz-Revilla C, et al. Renewed hope for a vaccine against the intestinal adult Taenia solium. J Parasitol. 2007; 93: 824-31.

12. Schantz PM, Moore AC, Munoz JL, et al. Neurocysticercosis in an Orthodox Jewish community in New York City. N Engl J Med. 1992; 327: 692-695.

13. Moore AC, Lutwick LI, Schantz PM, et al. Seroprevalence of cysticercosis in an Orthodox Jewish community. Am J Trop Med Hyg. 1995; 53: 439-442.

14. Morales-Montor J, Chavarria A, De León MA, et al. Host gender in parasitic infections of mammals: an evaluation of the female host supremacy paradigm. J Parasitol. 2004; 90: 531-546.

15. Escobedo G, Roberts CW, Carrero JC, et al. Parasite regulation by host hormones: an old mechanism of host exploitation? Trends Parasitol. 2005; 21: 588-593.

16. Bottasso O, Morales-Montor J. Neuroimmunomodulation during infectious diseases: mechanisms, causes and consequences for the host. Neuroimmunomodulation. 2009; 16: 65-67.

17. Roberts CW, Walker W, Alexander J. Sex-associated hormones and immunity to protozoan parasites. Clin Microbiol Rev. 2001; 14: 476-88.

18. Klein S. Hormonal and immunological mechanisms mediating sex differences in parasite infection. Parasite Immunol. 2004; 26: 247-64.

19. Morales J, Velasco T, Tovar V, et al. Castration and pregnancy of rural pigs significantly increase the prevalence of naturally acquired Taenia solium cysticercosis. Vet Parasitol. 2002; 108: 41-48.

20. Peña N, Morales J, Morales-Montor J, et al. Impact of naturally acquired Taenia solium cysticercosis on the hormonal levels of free ranging boars. Vet Parasitol. 2007; 21: 134-7.

21. Sarti E. La taeniasis y cisticercosis en México (revisión bibliográfica). Salud Pública Mex. 1986; 28: 556-563.

22. Flisser A. Teniosis and cysticercosis due to T. solium. Prog Clin Parasitol. 1994; 4: 77-116.

23. Organización Panamericana de la Salud. Epidemiología y control de la taeniosis y cisticercosis en América Latina. Washington, DC: OPS/OMS. 1994.

24. Chavarría A, Fleury A, García E, et al. Relationship between the clinical heterogeneity of neurocysticercosis and the immune-inflammatory profiles. Clin Immunol. 2005; 116: 271-8.

25. Larralde C, Morales J, Terrazas I, et al. Sex hormone changes induced by the parasite lead to feminization of the male host in murine Taenia crassiceps cysticercosis. J Steroid Biochem Mol Biol. 1995; 52: 575-80.

26. Huerta L, Terrazas LI, Sciutto E, et al. Immunological mediation of gonadal effects on experimental murine cysticercosis caused by Taenia crassiceps metacestodes. J Parasitol. 1992; 78: 471-6.

27. Vargas-Villavicencio JA, Larralde C, Morales-Montor J. Gonadectomy and progesterone treatment induce protection in murine cysticercosis. Parasite Immunol. 2006; 28: 667-74.

28. do Prado Júnior JC, Leal $\mathrm{M}$ de $\mathrm{P}$, Anselmo-Franci JA, et al. Influence of female gonadal hormones on the parasitemia of female Calomys callosus infected with the "Y" strain of Trypanosoma cruzi. Parasitol Res. 1998; 84: 100-5.

29. Soliman MF, Ibrahim MM. Antischistosomal action of atorvastatin alone and concurrently with medroxyprogesterone acetate on Schistosoma haematobium harboured in hamster: surface ultrastructure and parasitological study. Acta Trop. 2005; 93: $1-9$.

30. Nuñez GG, Costantino SN, Gentile T, et al. Immunoparasitological evaluation of Trichinella spiralis infection during human pregnancy: a small case series. Trans R Soc Trop Med Hyg. 2008 ; $102: 662-8$.
31. Kyurkchiev D, Ivanova-Todorova E, Kyurkchiev SD. New target cells of the immunomodulatory effects of progesterone. Reprod Biomed Online. 2010; 21: 304-11.

32. Zen M, Ghirardello A, Iaccarino L, et al. Hormones, immune response, and pregnancy in healthy women and SLE patients. Swiss Med Wkly. 2010; 140: 187-201.

33. Blois S, Zenclussen AC, Roux ME, et al. Asymmetric antibodies $(\mathrm{AAb})$ in the female reproductive tract. J Reprod Immunol. 2004; 64: 31-43.

34. Ivanova E, Kyurkchiev D, Altankova I, et al. CD83 monocyte-derived dendritic cells are present in human decidua and progesterone induces their differentiation in vitro. Am J Reprod Immunol. 2005; 53: 199-205.

35. Klein SL, Gamble HR, Nelson RJ. Role of steroid hormones in Trichinella spiralis infection among voles. Am J Physiol. 1999; 277: R1362-7.

36. Morales-Montor J, Baig S, Mitchell R, et al. Immunoendocrine interactions during chronic cysticercosis determine male mouse feminization: role of IL-6. J Immunol. 2001; 167: 4527-33.

37. Lezama-Dávila $\mathrm{CM}$, Isaac-Márquez $\mathrm{AP}$, Barbi J, et al. 17Beta-estradiol increases Leishmania mexicana killing in macrophages from DBA/2 mice by enhancing production of nitric oxide but not pro-inflammatory cytokines. Am J Trop Med Hyg. 2007; 76: 1125-7.

38. Santello FH, Del Vecchio Filipin M, Caetano LC, et al. Influence of melatonin therapy and orchiectomy on T cell subsets in male Wistar rats infected with Trypanosoma cruzi. J Pineal Res. 2009; 47: 271-6.

39. Morales-Montor J, Escobedo G, Rodríguez-Dorantes M, et al. Differential expression of AP-1 transcription factor genes c-fos and c-jun in the helminth parasites Taenia crassiceps and Taenia solium. Parasitology. 2004; 129: 233-243.

40. Escobedo G, Camacho-Arroyo I, Hernandez-Hernandez T, et al. Progesterone induces scolex evagination of the human parasite Taenia solium: evolutionary implications to the host-parasite relationship. J Biomed Biotechnol. 2010; 2010: 591079.

41. Camacho-Arroyo I, Guerra-Araiza C, Cerbon MA. Progesterone receptor isoforms are differentially regulated by sex steroids in the rat forebrain. Neuroreport. 1998; 9: 3993-399.

42. Butts CL, Shukair SA, Duncan KM, et al. Progesterone inhibits mature rat dendritic cells in a receptor-mediated fashion. Int Immunol. 2007; 19: 287-296.

43. Tait AS, Butts CL, Sternberg EM. The role of glucocorticoids and progestins in inflammatory, autoimmune, and infectious disease. J Leukoc Biol. 2008; 84: 924-31.

44. Szekeres-Bartho J, Polgar B. PIBF: The Double Edged Sword: Pregnancy and Tumor. Am J Reprod Immunol. 2010; 64: 77-86.

45. Vargas-Villavicencio JA, De León-Nava MA, Morales-Montor J. Immunoendocrine mechanisms associated with resistance or susceptibility to parasitic diseases during pregnancy. Neuroimmunomodulation. 2009; 16: 114-21.

46. León-Cabrera S, Cruz-Rivera M, Mendlovic F, et al. Standardization of an experimental model of human taeniosis for oral vaccination. Methods. 2009; 49: 346-50.

47. Hotez PJ, Brindley PJ, Bethony JM, et al. Helminth infections: the great neglected tropical diseases. J Clin Invest. 2008; 118: 1311-21.

48. Boussines M. Onchocerciasis control: biological research is still needed. Parasite. 2008; 15: 510-4.

49. Han ZG, Brindley PJ, Wang SY, et al. Schistosoma genomics: new perspectives on schistosome biology and host-parasite interaction. Annu Rev Genomics Hum Genet. 2009; 10: 211-40.

50. García HH, González AE, Del Bruto OH, et al. Strategies for the elimination of taeniasis/cysticercosis. J Neurol Sci. 2007; 15: 153-7. 
51. Kozak M, Kołodziej-Sobocińska M. Progress in the development of vaccines against helminthes. Wiad Parazytol. 2009; 55: 147-56.

52. Ortega CD, Ogawa NY, Rocha MS, et al. Helminthic diseases in the abdomen: an epidemiologic and radiologic overview. Radiographics. 2010; 30: 253-67.

53. Cruz-Revilla C, Toledo A, Rosas G, et al. Effective protection against experimental Taenia solium tapeworm infection in hamsters by primo-infection and by vaccination with recombinant or synthetic heterologous antigens. J Parasitol. 2006; 92: 864-7.

54. Daynes RA, Araneo BA. The development of effective vaccine adjuvants employing natural regulators of T-cell lymphokine production in vivo. Ann NY Acad Sci. 1994; 730: 144-161.

55. Danenberg HD, Ben-Yehunda A, Zakay-Rones Z, et al. Dehydroepiandrosterone (DHEA) treatment reverses the impaired immune response of old mice to influenza vaccination from influenza infection. Vaccine. 1995; 13: 1445-1448.

56. Evans T, Judd M, Dowell T, et al. The use of oral dehydroepiandrosterone sulfate as an adjuvant in tetanus and influenza vaccination of the elderly. Vaccine. 1996; 14: 1531-1537.

57. Burdick NC, Dominguez JA, Welsh TH Jr, et al. Oral administration of dehydroepiandrosterone-sulfate (DHEAS) increases in vitro lymphocyte function and improves in vivo response of pigs to immunization against keyhole limpet hemocyanin (KLH) and ovalbumin. Int Immunopharmacol. 2009; 9: 1342-6.

58. Bouman A, Heineman MJ, Faas MM. Sex hormones and the immune response in humans. Hum Reprod Update. 2005; 11: 411-23.

59. Stringer E, Antonsen E. Hormonal contraception and HIV disease progression. Clin Infect Dis. 2008; 47: 945-51.

60. Wira CR, Fahey JV, Ghosh M, et al. Sex Hormone Regulation of Innate Immunity in the Female Reproductive Tract: The Role of Epithelial Cells in Balancing Reproductive Potential with Protection against Sexually Transmitted Pathogens. Am J Reprod Immunol. 2010; 63: 544-565.

61. Wira CR, Grant-Tschudy KS, Crane-Godreau MA. Epithelial cells in the female reproductive tract: a central role as sentinels of immune protection. Am J Reprod Immunol. 2005; 53: 65-76.

62. Brabin L. Interactions of the female hormonal environment, susceptibility to viral infections, and disease progression. AIDS Patient Care STDS. 2002; 16: 211-21.

63. Avila G, Aguilar L, Romero-Valdovinos M, et al. Cytokine response in the intestinal mucosa of hamsters infected with Taenia solium. Ann N Y Acad Sci. 2008; 1149: 170-3.

64. Kasperska-Zajac A, Brzoza Z, Rogala B. Sex hormones and urticaria. J Dermatol Sci. 2008; 52: 79-86.

65. Mittman RJ, Bernstein DI, Steinberg DR, et al. Progesterone-responsive urticaria and eosinophilia. J Allergy Clin Immunol. 1989; 84: 304-10.

66. Zaitsu M, Narita S, Lambert KC, et al. Estradiol activates mast cells via a non-genomic estrogen receptor-alpha and calcium influx. Mol Immunol. 2007; 44: 1977-85.

67. Cocchiara R, Albeggiani G, Di Trapani G, et al. Oestradiol enhances in vitro the histamine release induced by embryonic histamine-releasing factor (EHRF) from uterine mast cells. Hum Reprod. 1992; 7: 1036-41.

68. Yang B, Zhou HJ, He QJ, et al. Termination of early pregnancy in the mouse, rat and hamster with DL111-IT and RU486. Contraception. 2000; 62: 211-6.

69. Gray GO, Leavitt WW. RU486 is not an antiprogestin in the hamster. J Steroid Biochem. 1987; 28: 493-7.

70. Grundmann O, Yoon SL. Irritable bowel syndrome: epidemiology, diagnosis and treatment: an update for health-care practitioners. J Gastroenterol Hepatol. 2010; 25: 691-9.

71. Ortiz DD. Chronic pelvic pain in women. Am Fam Physician. 2008; 77: 1535-42.
72. Myers B, Schulkin J, Greenwood-Van Meerveld B. Sex Steroids Localized To The Amygdala Increase Pain Responses To Visceral Stimulation In Rats. J Pain. 2011; 12: 486-494.

73. Heitkemper MM, Chang L. Do fluctuations in ovarian hormones affect gastrointestinal symptoms in women with irritable bowel syndrome? Gend Med. 2009; 6: 152-67.

74. Chang L, Heitkemper MM. Gender differences in irritable bowel syndrome. Gastroenterology. 2002; 123: 1686-701.

75. Chen G, Shi JX, Qi M, et al. Effects of progesterone on intestinal inflammatory response, mucosa structure alterations, and apoptosis following traumatic brain injury in male rats. J Surg Res. 2008; 147: 92-8.

76. Freedman ND, Lacey JV Jr, Hollenbeck AR, et al. The association of menstrual and reproductive factors with upper gastrointestinal tract cancers in the NIH-AARP cohort. Cancer. 2010; 116: 1572-81.

77. Tran A, Villeneuve JP, Bilodeau M, et al. Treatment of chronic bleeding from gastric antral vascular ectasia (GAVE) with estrogen-progesterone in cirrhotic patients: an open pilot study. Am J Gastroenterol. 1999; 94: 2909-11.

78. Olsson R, Mattsson LA, Obrant K, et al. Estrogen-progestogen therapy for low bone mineral density in primary biliary cirrhosis. Liver. 1999; 19: 188-92.

79. Saqui-Salces M, Rocha-Gutiérrez BL, Barrios-Payán JA, et al. Effects of estradiol and progesterone on gastric mucosal response to early Helicobacter pylori infection in female gerbils. Helicobacter. 2006; 11: 123-30.

80. Montoneri C, Drago F. Effects of pregnancy in rats on cysteamine-induced peptic ulcers: role of progesterone. Dig Dis Sci. 1997; 42: 2572-5. 